\title{
A Note on the Mathematical Model for the Estimation of Fishing Rate of Mojako by Means of Tagging Experiments on Drifting Seaweeds
}

\author{
Syoiti TANAKA* \\ (Received August 7, 1971)
}

\begin{abstract}
The basic model for the number of tags $N$ presented in the previous paper is modified, in order to take into account the fact that tags shed from seaweeds are not vulnerable to the fishery, and hence the coefficient of fishing ${ }_{\kappa} F$ can be applied only to retained tags $N_{T}$, but not to the total tags $N$. The results from a modified model are compared with those from the previous one. It reveals that the results from the latter, which is very simple in expression, is a good approximation to that from the former and the error involved in the estimate of the coefficient of tag shedding $M$ based on the previous model is thought to be negligible. This modification of the model does not affect the estimates of other parameters such as ${ }_{\kappa} F$.
\end{abstract}

An attempt has been made by the author ${ }^{11}$ to assess the fishing rate of mojako (juvenile yellowtail, Seriola quinqueradiata) from data of tagging experiments on drifting seaweeds, and based on a mathematical model for the number of tags $N$

$$
\frac{d N}{d t}=-(D+\kappa F) N
$$

Here, $D$ is the coefficient of decrease due to drifting away from the area of release, $F$, the coefficient of fishing by mojako fishery and $\kappa$, the rate of discovery of recaptured tags. It should be pointed out, however, that this model is not totally correct. As there is almost no possibility of shed tags to be recaptured by the mojako fishery, the decrease of tags due to fishing should be applied not to the entire tags of $N$, but only to the tags retained by seaweeds, $N_{T}$. Therefore, the term $-\kappa F N$ on the right hand side of (1) should be replaced by $-\kappa F N_{T}$. This modification is particularly important when the coefficient of tag shedding is to be estimated from coastal recoveries ${ }^{3)}$. Nevertheless, it is expected that the model provides a fairly good approximation to a more exact model and the solution is simple and convenient for further analyses.

The purpose of this paper is to consider a more exact model and to examine the validity of (1) as an approximation.

\section{Development of Formulae}

The basic model for $N$ given by (1) is now replaced by a new model of

$$
\frac{d N}{d t}=-D N-\kappa F N_{T}
$$

while the model for $N_{T}$ is the same as Formula (2) in the previous paper ${ }^{1)}$, which is

\footnotetext{
* Ocean Research Institute, University of Tokyo, Nakano, Tokyo.（田中冒一：東京大学海洋研究所)
} 


$$
\frac{d N_{T}}{d t}=-(D+\kappa F+M) N_{T} .
$$

Here, $M$ is the coefficient of tag shedding from drifting seaweeds. Substituting the solution of (3) into (2), a new equation

$$
\frac{d N}{d t}+D N=-\kappa F N_{0} e^{-(D+\kappa F+M \mid t}
$$

is obtained. The solution of (4) is

$$
N=N_{0} e^{-D t}\left(\frac{M}{\kappa F+M}+\frac{\kappa F}{\kappa F+M} e^{-\langle\kappa F+M) t}\right)
$$

or

$$
N=N_{0} e^{-D t}\left(1-\frac{\kappa F}{\kappa F+M}\left(1-e^{-\kappa(F+M \mid t}\right)\right) .
$$

On the other hand, the solution derived from (1) is

$$
N_{(1)}=N_{0} e^{-\{D+\kappa F\} t} \text {. }
$$

The modification of the basic model from (1) to (2) would also cause change of $n$, the number of tags drifted away from the area of release but still being drifted about in the vicinity. From Formula (4) of the previous paper $^{1 \text { ) }}$,

$$
\frac{d n}{d t}=D N-(H+L) n,
$$

where $H$ is the coefficient of decrease due to drifting ashore, and $L$, the coefficient of complete loss from the study area due to drifting. Substitution of (5) into (7) will bring an equation for $n$,

$$
\frac{d n}{d t}+(H+L) n=D N_{0} e^{-D t}\left(\frac{M}{\kappa F+M}+\frac{\kappa F}{\kappa F+M} e^{-(\kappa F+M) t}\right),
$$

which will result in

$$
\begin{aligned}
n= & \frac{M}{\kappa F+M} \frac{D N_{0}}{D-H-L}\left(e^{-(H+L) t}-e^{-D t}\right) \\
& +\frac{\kappa F}{\kappa F+M} \frac{D N_{0}}{D+\kappa F+M-H}=L^{-\left(e^{-(H+L) t}-e^{-(D+\kappa F+M) t}\right),}
\end{aligned}
$$

or

$$
\begin{aligned}
n= & \frac{D N_{0}}{D-H-L}\left(e^{-(H+L) t}-e^{-D t}\right)-\frac{\kappa F}{\kappa F+M}\left(\frac{D N_{0}}{D-H-L}\left(e^{-(\not L+L) t}-e^{-D t}\right)\right. \\
& \left.-\frac{D N_{0}}{D+\kappa F+M-H-L}\left(e^{-(H+L) t}-e^{-(D+\kappa F+M) t}\right)\right) .
\end{aligned}
$$

The corresponding result from (1) and (7) is

$$
n_{(1)}=\frac{D N_{0}}{D+\kappa \bar{F}-H-L}\left(e^{-(H+L) t}-e^{-(D+\kappa F) t}\right) .
$$

The proportion of the retained tags based on (1) is simple. For the tags in the area of release, 


$$
N_{T} / N_{(1)}=e^{-m t}
$$

and for the tags drifted away from the area of release,

$$
n_{T} / n_{(1)}=e^{-M t} \text {. }
$$

Here $n_{T}$ is the number of retained tags among $n$. On the contrary, the proportion based on (2) is complicated. For the tags in the area of release, for instanse,

$$
N_{T} / N=e^{-(\kappa F+M) t} /\left(1-\frac{\kappa F}{\kappa F+M}\left(1-e^{-(\kappa F+M) t}\right)\right) .
$$

\section{Accuracy of the Approximate Model}

According to TANAKA's paper ${ }^{2}$, the estimated values of the various parameters are as follows; $D$ is generally larger than $0.1 /$ day, most of values of $\kappa F$ are in a range of $0.02-$ $0.03 /$ day, and $M$ is approximately $0.02 /$ day. It is evident that $\kappa F$ and $M$ are much smaller than $D$. Taking this fact into consideration, for moderate values of $t$

$$
1-\frac{\kappa F}{\kappa F+M}\left(1-e^{-\kappa F+M / t}\right) \risingdotseq e^{-\kappa F t}
$$

and hence,

$$
N \risingdotseq N_{(1)} \text {. }
$$

Taking the results for the tag releases off Tokushima Prefecture in 1964 for example, that is $\kappa F=0.026$ and $M=0.02^{11}$, the left and right hand sides of (14) are compared in Table 1. Upto $t=20$ days, the error is less than $10 \%$, but it begins to increase sharply beyond this point. Tag recoveries by the mojako fishery in 1964-65 amounted to 124 tags among which 105 or $85 \%$ were recovered not later than 20 days after release. It may be said that $N_{(1)}$ is a reasonable approximation of $N$.

As the value of $n$ is influenced dominantly by large values of $N$, that is $N$ at small $t$, it is expected that the approximation of $n_{(1)}$ to $n$ is better than that of $N_{(1)}$. The values of $D=0.113$ and $H+L=0.022$ cited from the same example, together with the other two values mentioned before, would give the ratio of $n_{(1)}$ to $n$ at the limit when $t$ increases infinitely,

$$
n_{(1)} / n=\frac{1 /(D+\kappa F-H-L)}{M /(\kappa F+M)(D-H-L)+\kappa F /(\kappa F+M)(D+\kappa F+M-H-L)}=0.96 .
$$

This suggests that the error of $n_{(1)}$ is almost negligible. Although the accuracy depends upon the values of parameters, it may be concluded that the error of $n_{(1)}$ is very small particularly when the number of tags recovered by the mojako fishery in the offshore area is small.

All the parameters except $M$ are estimated from the recoveries of retained tags and are free from the error in the model of (1). For the estimation of $M$ in which the ratio among recoveries between retained tags and shed tags is utilized ${ }^{2,3)}, n_{(1)} \risingdotseq n$ means that 
Table 1. Accuracy of $N_{\{1\}}$ as an approximation to $N$.

\begin{tabular}{|c|c|c|c|c|c|c|c|}
\hline$t$ (days) & 1 & 5 & 10 & 20 & 30 & 40 & $\infty$ \\
\hline $1-\frac{\kappa F}{\kappa F+M}\left(1-e^{-(\kappa F+M(N) t)}\right.$ & 0.9746 & 0.8839 & 0.7916 & 0.6600 & 0.5770 & 0.5246 & 0.4348 \\
\hline$e^{-k F T}$ & 0.9743 & 0.8781 & 0.7711 & 0.5945 & 0.4584 & 0.3535 & 0 \\
\hline$N_{(1)} / N$ & 0.9997 & 0.9934 & 0.9741 & 0.9008 & 0.7945 & 0.6738 & 0 \\
\hline
\end{tabular}

$$
n_{T} / n \doteqdot n_{T} / n_{(1)}=e^{-M t}
$$

The validity of the estimates of $M$ based on the model of (1) is considered to be well demonstrated.

\section{References}

1) S. TANAKA: This Bull., 33, 1108-1115 (1967).

2) S. TANAKA: ibid., 34, 775-780 (1968).

3) S. TANAKA: ibid., 37, 1067-1072 (1971). 